\title{
Effects of Breast Cancer Treatment on Shoulder Function: What to Expect and How to Treat?
}

Neto $\mathbf{C M}^{{ }^{*}}$, Pezarat $\mathbf{P}^{2}$ and Oliveira $\mathbf{R}^{2}$

${ }^{1}$ Hospital Prof. Dr. Fernando Fonseca, Amadora, 2720-276, Portugal

${ }^{2}$ Faculdade de Motricidade Humana, Universidade de Lisboa, Lisboa, 1649-004, Portugal

\section{Abstract}

Breast cancer treatment may lead to side effects such as shoulder pain, restricted shoulder mobility, fibrosis, breast cancer-related lymphedema, and anatomical and biomechanical changes of the shoulder, which will contribute to functional status limitations. Function of the upper limb requires adequate mobility of the shoulder, including the scapula, and an efficient neuromuscular coordination. Movement deviation patterns in women following surgery for breast cancer are similar to those seen in other known shoulder conditions. Exercise therapy and scapular stabilization exercises were found to be an effective approach for controlling pain, promote normal motor control and decreasing disability. The main purpose of the present paper is to review the shoulder movement dysfunctions after breast cancer treatment and to briefly characterize the main physical therapy intervention strategies to treat or prevent these dysfunctions.

\section{Introduction}

Cancer rehabilitation is a process that helps cancer survivors achieve and maintain the highest possible physical, social, psychological, and vocational functioning, within the limits created by cancer and its treatments [1].

Among women, breast cancer is the most commonly diagnosed cancer and the second leading cause of cancer death [2]. However, due to new treatments and early detection, breast cancer survival has improved over time [2]. Breast cancer treatment may lead to side effects such as shoulder pain, restricted shoulder mobility, fibrosis, breast cancer-related lymphedema [3-5], and anatomical and biomechanical changes of the shoulder [6], which will contribute to functional status limitations [7-9]. These disorders may manifest immediately after treatment of breast cancer or may present many years later [10]. Function of the upper limb requires adequate mobility of the shoulder including the scapula, and an efficient neuromuscular coordination. Alterations of the scapulohumeral rhythm will cause microtrauma and long-term pain. It is suggested that subtle changes in this space may result in compression of the subacromial structures such as the bursa and rotator cuff during arm elevation [11]. The glenohumeral (GU) is a very mobile joint, and its stability depends largely on motor control. In this way the evaluation of this control and the directed treatment to achieve improvement should be a central part of the physical therapy approach [12]. Several types of functionally oriented exercises have been developed based on scapulohumeral kinematics and the kinetic chain beyond the shoulder range of motion (ROM) and strength [13]. Counseling exercises and scapula-oriented shoulder exercises may be effective with regard to pain and quality of life [14] as well improving shoulder mobility [15].

The main purpose of the present paper is to review the shoulder movement dysfunctions after breast cancer treatment and to briefly characterize the main physical therapy intervention strategies to treat these dysfunctions.

\section{Shoulder Dysfunction after Breast Cancer Treatment}

Women treated for breast cancer often complain of shoulder pain and decreased function post-surgery; $10-55 \%$ of women show restricted glenohumeral range of movement, 22-38\% complains of shoulder pain, and $42-56 \%$ report difficulties with lifting the upper limb [16]. The surgical incisions through the skin and fasciae will result in scar tissue formation in the anterior chest wall region. This scar tissue may impair normal gliding between skin related structures, fasciae, and muscles that are necessary for freeand normal motion of the shoulder girdle. That, associated with pain, protective postures and fear of complications, will promote a resting shoulder girdle mal-alignment and a decreased shoulder motion [17]. Soft tissue fibrosis secondary to radiation therapy would likely exacerbate decreased tissue flexibility and motion restrictions [18]. Pectoralis minor and major muscles lengths are believed to have an important effect on shoulder resting position [19].

Glenohumeral (GU) joint is the most mobile joint that relies heavily on mid-range stability on muscle control [12]. Arm elevation depends on interaction and coordination of both glenohumeral and scapulothoracic movements which ensure that functional activities can occur without the head of the humerus impacting on the coracoacromial arch and placing the soft tissue structures across the shoulder joint, in risk of impingement. The absence of osseous stability at the GU joint means that the shoulder complex relies on the interaction of both static and dynamic structures to provide joint stability [20]. Muscles of the shoulder form the dynamic structures and can be divided functionally into stabilizers and prime movers [21]. Timed interaction between these two groups of muscles is essential to achieve a smooth and physiologic scapulohumeral rhythm [22-24].

Considering the kinematics of shoulder movement, shoulder tightness affects glenohumeral translation and scapulohumeral rhythm,

"Corresponding Author: Dr. Carla Martinho Neto, PT in Hospital Prof. Dr. Fernando Fonseca, Amadora, Portugal and $\mathrm{PhD}$ student in Faculdade de Motricidade Humana-Universidade de Lisboa, Portugal; E-mail: carla.martinho.neto@gmail.com

Citation: Neto CM, Pezarat P, Oliveira R (2018) Effects of Breast Cancer Treatment on Shoulder Function: What to Expect and How to Treat? Int J Phys Ther Rehab 4 147. doi: https://doi.org/10.15344/2455-7498/2018/147

Copyright: (C) 2018 Neto et al. This is an open-access article distributed under the terms of the Creative Commons Attribution License, which permits unrestricted use, distribution, and reproduction in any medium, provided the original author and source are credited. 
followed by changes in scapular motion $[25,26]$. In particular, shortened pectoralis major and minor covering the anterior part of the shoulder and chest can impose restrictions on upward rotation, external rotation, posterior tilt and elevation of the scapula [27] with changes for the scapulohumeral rhythm.

Some studies have highlighted, but have not quantified, scapula dyskinesia (e.g. winging of the scapula) in patients demonstrating limited shoulder movements. Its incidence varies widely between $0 \%$ and $74.7 \%$ [28-33]. Evaluation of the shoulder dysfunction in breast cancer patients have been based in clinical examination, including range of movement measures and functional activities. This might condition in which the medial border and inferior angle of the scapula protrudes prominently from the thorax. It can arise from numerous pathologic processes, which lead to a deficiency of the muscles that play a central role in pulling the scapula towards the thoracic wall, like serratus anterior [34]. Post-operative pain, length and tightness of scars, neural damage and muscle atrophy, might be some of the causes [28]. Clinical observations have been reported for muscle morbidity of pectoralis major, serratus anterior and latissimus dorsi $[28,29]$.

Shamley et al. suggests alterations to the normal biomechanics of the shoulder complex in patients treated for breast cancer. They found an increased internal rotation of the scapula during elevation after surgery [35], supporting a real connection between soft tissue alterations and biomechanical changes at the shoulder. Borstad et al. [36] found the same changes after only two months of breast cancer surgery. Decreased scapula upward rotation and posterior tilting, and increased scapula internal rotation have all been associated with subacromial impingement syndrome [37]. Other studies [38,39] showed an increased upward rotation of the scapula following mastectomy for breast cancer. It is believed that this represents a compensatory mechanism for a dysfunctional motion of the GU joint. These results were similar in symptomatic [38] and asymptomatic [39] women in relation to shoulder pain.

Shamley et al. [40] found a decrease in the size of the pectoralis major and minor muscles and a decreased EMG activity in upper trapezius and rhomboid muscles during arm elevation, in contrast to during a low-load upper limb functional task [41] and arm elevation [33]. Less activity in the serratus anterior and increased activity in the deltoid was also found in breast cancer patients [33]. These findings suggest that motor control impairments in the shoulder complex, which may persist for a long time, play an important role in the development of shoulder pain and dysfunction in breast cancer survivors. An association between altered muscle activity at the shoulder and functional limitation was also found in this population for up to 6 years after treatment [40].

Regaining upper-body function after breast cancer surgery seems to take significantly longer than women's own or general expectations. Many women reported difficulties in simple day-to-day activities, such as, driving, sleeping, housework and gardening in addition to a reduced ability to do their usual occupations [42]. In a longitudinal investigation designed to assess the physical and psychosocial recovery of women6 months following breast cancer, the range in the function/symptom scale - Disability of the Arm, Shoulder and Hand questionnaire (DASH) scores was $0-72$, with approximately $50 \%$ of the group reporting upper-body function scores of 11 or lower, explain the high variability of these findings. Scapular winging is a and lowering of the arm, with significant pain levels for up to 6 years other authors, who found an increased activity of the upper trapezius

representing 'good' arm function [43]. Continuous upper-body problems, and therefore difficulties when performing everyday tasks, existed for many women after 1 year post-surgery [42].

Shoulder neuromuscular impairments commonly experience by breast cancer survivors, contribute to the development of shoulder pain and dysfunction. Given that the majority of these impairments can be addressed with appropriate rehabilitation interventions, it is crucial that physical therapists better understand these potential causes in order to develop specific evidence-based intervention programs to treat and prevent shoulder pain and dysfunction in breast cancer survivors.

\section{Rehabilitation of Shoulder Function in Breast Cancer Survivors}

The shoulder is a very mobile joint that relies heavily on mid-range stability on muscle control. Therefore, evaluation of such control and treatment directed at its improvement should form an integral part of management of all shoulder disorders [12]. The scapula has a major and pivotal role in normal shoulder function. Its position and motion provide the parameters to allow normal physiology and biomechanics of the shoulder to occur [22].

There is evidence of scapular kinematic alterations associated with shoulder and neck pain [44]. There is also evidence of altered scapular muscle recruitment patterns in these patients, with respect to changes in strength, flexibility, motor control and timing of the surrounding muscles [44]. In particular, lack of flexibility, in combination with muscle dysfunction of serratus anterior and lower trapezius, may be the clinical base for a treatment program given to these patients. The available evidence in clinical trials supports the use of therapeutic exercise in the rehabilitation of these patients [44]. In patients diagnosed with subacromial impingement syndrome and accompanying scapular dyskinesia, exercise therapy was found to be an effective approach for controlling pain and decreasing disability. However, adding scapular stabilization exercises to the exercise program produced slightly better results regarding scapular kinematics after 6 and 12 weeks of training [45].

From our knowledge until now, there is only one published study that has developed a scapula-oriented shoulder exercise program on upper limb dysfunction in breast cancer survivors, and compared with a general exercise and a non-exercise group. Their findings showed that pain, physical function, social function and quality of life improved significantly, and upper limb disabilities, range of motion and strength of the shoulder external rotation showed trends towards significance after the scapula-oriented exercise compared with baseline. The improvement in global quality of life and external shoulder rotation strength were significantly greater in the scapulaoriented exercise program than in the general exercise and control groups [14].

Other authors have studied the effects of general upper limb exercise interventions in this population. A systematic review on exercise interventions for upper limb dysfunction due to breast cancer treatment, has found that upper limb exercise (shoulder ROM and stretching) is helpful in recovering upper limb function following surgery for breast cancer; structured upper limb exercise program shows greater benefit in restoration of shoulder ROM; and exercise programs benefit quality of life and upper extremity strength through resistance exercise [46]. A structured weekly resistance training and 
Citation: Neto CM, Pezarat P, Oliveira R (2018) Effects of Breast Cancer Treatment on Shoulder Function: What to Expect and How to Treat? Int J Phys Ther Rehab 4: 147. doi: https://doi.org/10.15344/2455-7498/2018/147

Page 3 of 4

stretching program improved ROM in both forward flexion and abduction and strength in abduction plane, and did not precipitate lymphedema [47]. Cavanaugh [48] discuss the findings of four studies in which implementation of early exercise and activity after axillary node dissection was investigated. The results of this reviewed studies support the implementation of this approach, as opposed to restriction after axillary lymph node dissection.

Although exercise interventions have a positive effect on quality of life in cancer patients [49], in physical and psychosocial areas [50], have been associated with a lesser symptom experience for shoulder limitations, muscular chest wall pain, weight gain, lymphedema, and breathlessness [51].

Movement deviation patterns in women following surgery for breast cancer are similar to those seen in other known shoulder conditions [6]. Therefore we strongly recommend that the breast cancer patient's rehabilitation program should include a scapular motor control based approach.

One of the goals of any motor control rehabilitation is to gain awareness of, and the ability to activate the deep stabilizers of the region prior to activation of the, usually, more superficial torque prime movers and to maintain that activation during activity. Another is retraining of optimal movement patterns. Both involve motor program retraining and therefore, refined, controlled activation of the deep stabilizing force couples, using either strategies of isolation or controlled posture or movement facilitated by imagery [12].

Cools et al. [44] have suggested practical guidelines for the rehabilitation of scapular dyskinesia in patients with chronic complaints in the upper quadrant:

1. Conscious muscle control of the scapular muscles: In the early stage of scapular training, conscious muscle control of the scapular muscles may be necessary to improve proprioception and to normalize scapular resting position.

2. Muscle (re) training: Depending on the clinical examination results, the therapist may decide in the second stage of scapular muscle training to focus more on muscle control and cocontraction (advanced control during basic activities) or muscle strength. Scapular co-contraction may be trained in basic positions, movements and exercises. As the shoulder girdle works in both open and closed-chain activities, the muscles should be trained to respond to both situations, by challenging the maintenance of the new scapular position under load, using weight-bearing and non-weight-bearing tasks of the upper limb. These should be consistent with the functional needs of the patient.

3. General scapular strengthening exercises: Once muscle balance is restored, in the third stage of scapular muscle rehabilitation program, general scapular strengthening exercises may be used to increase muscle strength [44].

A common recommendation in rehabilitation is to limit the amount of weight used during glenohumeral and scapulothoracic exercises to assure that the appropriate muscles are being utilized and not larger compensatory muscles [52].

There is evidence that exercise and physical activity are effective, well-tolerated and rewarding complementary interventions to enhance the quality of life in women with breast cancer. Physical therapy have an important role in preventing further upper limb decline and restore function in breast cancer survivors.

\section{Key Clinical Points}

1. Breast cancer treatment may lead to side effects which will contribute to anatomical and biomechanical changes of the shoulder and functional limitations that may persist for many years;

2. Movement deviation patterns in women following surgery for breast cancer are similar to those seen in other known shoulder conditions- scapula dyskinesia - which means a motor control impairments in the shoulder complex;

3. Breast cancer patient's rehabilitation program should include a scapular motor control based approach.

\section{Competing Interests}

The authors declare that they have no competing interests.

\section{References}

1. Ganz PA (1990) Current issues in cancer rehabilitation. Cancer 65: 742-751.

2. Siegel RL, Miller KD, Jemal A (2018) Cancer Statistics 2018. Ca Cancer J Clin 68: 7-30.

3. Johansen S, Fosså K, Nesvold IL, Malinen E, Fosså SD, et al. (2014) Arm and shoulder morbidity following surgery and radiotherapy for breast cancer. Acta Oncol 53: 521-529.

4. Hidding JT, Beurskens $\mathrm{CH}$, van der Wees PJ, van Laarhoven HW, Nijhuis-van der Sanden MW, et al. (2014) Treatment related impairments in arm and shoulder in patients with breast cancer: a systematic review. PLoS One 9 : e96748.

5. Kootstra JJ, Hoekstra-Weebers JE, Rietman JS, de Vries J, Baas PC, et al. (2010) A longitudinal comparison of arm morbidity in stage I-II breast cancer patients treated with sentinel lymph node biopsy, sentinel lymph node biopsy followed by completion lymph node dissection, or axillary lymph node dissection. Ann Surg Oncol 17: 2384-2394.

6. Shamley D, Lascurain-Aguirrebeña I, Oskrochi R (2014) Clinical anatomy of the shoulder after treatment for breast cancer. Clin Anat 27: 467-477.

7. Boquiren VM, Hack TF, Thomas RL, Towers A, Kwan WB, et al. (2016) A longitudinal analysis of chronic arm morbidity following breast cancer surgery. Breast Cancer Res Treat 157: 413-425.

8. Stubblefield MD, Keole N (2014) Upper body pain and functional disorders in patients with breast cancer. PM R 6: 170-183.

9. Ernst MF, Voogd AC, Balder W, Klinkenbijl JH, Roukema JA, et al. (2002) Early and late morbidity associated with axillary levels I-III dissection in breast cancer. J Surg Oncol 79: 151-155.

10. Hayes SC, Johansson K, Stout NL, Prosnitz R, Armer JM, et al. (2012) Upperbody morbidity after breast cancer: incidence and evidence for evaluation, prevention, and management within a prospective surveillance model of care. Cancer 118: 2237-2249.

11. Michener LA, McClure PW, Karduna AR (2003) Anatomical and biomechanical mechanisms of subacromial impingement syndrome. Clin Biomech 18: 369-379.

12. Magarey $M E$, Jones MA (2003) Dynamic evaluation and early management of altered motor control around the shoulder complex. Man Ther 8: 195206.

13. Markes M, Brockow T, Resch KL (2006) Exercise for women receiving adjuvant therapy for breast cancer. Cochrane Database Syst Rev 18: CD005001.

14. Lee SA, Kang JY, Kim YD, An AR, Kim SW, et al. (2010) Effects of a scapulaoriented shoulder exercise programme on upper limb dysfunction in breast cancer survivors: a randomized controlled pilot trial. Clin Rehabil 24: 600613. 
Citation: Neto CM, Pezarat P, Oliveira R (2018) Effects of Breast Cancer Treatment on Shoulder Function: What to Expect and How to Treat? Int J Phys Ther Rehab 4: 147. doi: https://doi.org/10.15344/2455-7498/2018/147

Page 4 of 4

15. D'Egidio V, Sestili C, Mancino M, Sciarra I, Cocchiara R, et al. (2017) Counseling interventions delivered in women with breast cancer to improve health-related quality of life: a systematic review. Qual Life Res 26: 2573 2592.

16. McNeely ML, Campbell K, Ospina M, Rowe BH, Dabbs K, et al. (2010) Exercise interventions for upper-limb dysfunction due to breast cancer treatment. Cochrane Database Syst Rev 16: CD005211.

17. Kärki A, Simonen R, Mälkiä E, Selfe J (2005) Impairments, activity limitations and participation restrictions 6 and 12 months after breast cancer operation. J Rehabil Med 37: 180-188.

18. Riekki R, Harvima IT, Jukkola A, Risteli J, Oikarinen A, et al. (2004) The production of collagen and the activity of mast-cell chymase increase in human skin after irradiation therapy. Exp Dermatol 13: 364-371.

19. Borstad JD (2006) Resting position variables at the shoulder: evidence to support a posture-impairment association. Phys Ther 86: 549-557.

20. Phadke V, Camargo P, Ludewig P (2009) Scapular and rotator cuff muscle activity during arm elevation: A review of normal function and alterations with shoulder impingement. Rev Bras Fisioter 13: 1-9.

21. Comerford MJ, Mottram SL (2001) Movement and stability dysfunction-contemporary developments. Man Ther 6: 15-26.

22. Kibler WB (1998) The role of the scapula in athletic shoulder function. Am Sports Med 26: 325-37.

23. Sahrmann SA (2002) Diagnosis and Treatment of Movement Impairment Syndromes. St Louis Missouri: Mosby.

24. Hess SA (2000) Functional stability of the glenohumeral joint. Man Ther 5: 63-71.

25. Lin JJ, Lim HK, Yang JL (2006) Effect of shoulder tightness on glenohumeral translation, scapular kinematics, and scapulohumeral rhythm in subjects with stiff shoulders. J Orthop Res 24: 1044-1051.

26. Rundquist PJ (2007) Alterations in scapular kinematics in subjects with idiopathic loss of shoulder range of motion. J Orthop Sports Phys Ther 37: 19-25

27. Borstad JD, Ludewig PM (2005) The effect of long versus short pectoralis minor resting length on scapular kinematics in healthy individuals. J Orthop Sports Phys Ther 35: 227-238.

28. Gutman H, Kersz T, Barzilai T, Haddad M, Reiss R, et al. (1990) Achievements of physical therapy in patients after modified radical mastectomy compared with quadrantectomy, axillary dissection, and radiation for carcinoma of the breast. Arch Surg 125: 389-391.

29. Gerber L, Lampert M, Wood C, Duncan M, D'Angelo T, et al. (1992) Comparison of pain, motion, and edema after modified radical mastectomy vs. local excision with axillary dissection and radiation. Breast Cancer Res Treat 21: 139-145.

30. Adriaenssens $N$, De Ridder $M$, Lievens $P$, Van Parijs $H$, Vanhoeij $M$, et al (2012) Scapula alata in early breast cancer patients enrolled in a randomized clinical trial of post-surgery short-course image-guided radiotherapy. World J Surg Oncol 10: 86.

31. Rizzi SK, Haddad CA, Giron PS, Pinheiro TL, Nazário AC, et al. (2016) Winged scapula incidence and upper limb morbidity after surgery for breast cancer with axillary dissection. Support Care Cancer 24: 2707-2715

32. Teixeira LFN, Lohsiriwat V, Schorr MC, Luini A, Galimberti V, et al. (2014) Incidence, Predictive Factors, and Prognosis for Winged Scapula in Breast Cancer Patients, after Axillary Dissection. Support Care Cancer 22: 16111617.

33. Pereira TB, Bergmann A, Ribeiro AC, Da Silva JG, Dias R, et al. (2009) Myoeletric activity pattern of scapular muscles after axillary lymphadenectomy in breast cancer. Rev Bras Ginecol Obstet 31: 224-229.

34. Martin RM, Fish DE (2008) Scapular winging: anatomical review, diagnosis, and treatments. Curr Rev Musculoskelet Med 1: 1-11.

35. Shamley D, Srinaganathan R, Oskrochi R, Lascurain-Aguirrebeña I, Sugden $\mathrm{E}$, et al. (2009) Three-dimensional scapulothoracic motion following treatment for breast cancer. Breast Cancer Res Treat 118: 315-322.

36. Borstad JD, Szucs KA (2012) Three-dimensional scapula kinematics and shoulder function examined before and after surgical treatment for breast cancer. Hum Mov Sci 31: 408-418.

37. Ludewig PM, Reynolds JF (2009) The association of scapular kinematics and glenohumeral joint pathologies. J Orthop Sports Phys Ther 39: 90-104.
38. Shamley D, Lascurain-Aguirrebeña I, Oskrochi R, Srinaganathan R (2012) Shoulder morbidity after treatment for breast cancer is bilateral and greater after mastectomy. Acta Oncol 51: 1045-1053.

39. Crosbie J, Kilbreath SL, Dylke E, Refshauge KM, Nicholson LL, et al. (2010) Effects of mastectomy on shoulder and spinal kinematics during bilateral upper-limb movement. Phys Ther 90: 679-692.

40. Shamley DR, Srinanaganathan R, Weatherall R, Oskrochi R, Watson M, et al. (2007) Changes in shoulder muscle size and activity following treatment for breast cancer. Breast Cancer Res Treat 106: 19-27.

41. Galiano-Castillo N, Fernández-Lao C, Cantarero-Villanueva I, Fernándezde-Las-Peñas C, Menjón-Beltrán S, et al. (2011) Altered pattern of cervical muscle activation during performance of a functional upper limb task in breast cancer survivors. Am J Phys Med Rehabil 90: 349-355.

42. Collins LG, Nash R, Round T, Newman B (2004) Perceptions of upper-body problems during recovery from breast cancer treatment. Support Care Cancer 12: 106-113.

43. Hayes S, Battistutta D, Newman B (2005) Objective and subjective upper body function six months following diagnosis of breast cancer. Breast Cancer Res Treat 94: 1-10.

44. Cools AM, Struyf F, De Mey K, Maenhout A, Castelein B, et al. (2014) Rehabilitation of scapular dyskinesis: from the office worker to the elite overhead athlete. Br J Sports Med 94: 692-627.

45. Turgut E, Duzgun I, Baltaci G (2017) Effects of Scapular Stabilization Exercise Training on Scapular Kinematics, Disability, and Pain in Subacromial Impingement: A Randomized Controlled Trial. Arch Phys Med Rehabil 98: 1915-1923.

46. Galantino ML, Stout NL (2013) Exercise interventions for upper limb dysfunction due to breast cancer treatment. Phys Ther 93: 1291-1297.

47. Kilbreath SL, Refshauge KM, Beith JM, Ward LC, Lee M, et al. (2012) Upper limb progressive resistance training and stretching exercises following surgery for early breast cancer: a randomized controlled trial. Breast Cancer Res Treat 133: 667-676.

48. Cavanaugh KM (2011) Effects of early exercise on the development of lymphedema in patients with breast cancer treated with axillary lymph node dissection. J Oncol Pract 7: 89-93.

49. Ferrer RA, Huedo-Medina TB, Johnson BT, Ryan S, Pescatello LS, et al. (2011) Exercise interventions for cancer survivors: a meta-analysis of quality of life outcomes. Ann Behav Med 41: 32-47.

50. Hwang JH, Chang HJ, Shim YH, Park WH, Park W, et al. (2008) Effects of supervised exercise therapy in patients receiving radiotherapy for breast cancer. Yonsei Med J 49: 443-450. 